\begin{tabular}{|l|l|}
\hline & \\
\hline
\end{tabular}

RESEÑA

\title{
Bourdieu en el campo educativo argentino (Amar, H. M., 2016) Editorial Biblos
}

\author{
Bourdieu no campo educativo argentino (Amar. H. M., 2016) - Editora Biblos \\ Bourdieu in the Argentine educational field (Amar. H. M., 2016) - Biblos Press
}

\section{Iván Surge ${ }^{1}$}

orcid.org/0000-0003-4052-4768 ivansurge@yahoo.com.ar

Recebido em: 14 jan. 2018 Aprovado em: 19 jul. 2020. Publicado em: 2 dez. . 2020.

\section{(c) (1)}

Artigo está licenciado sob forma de uma licença Creative Commons Atribuição 4.0 Internacional.

\section{Resumen}

Este libro es fruto de la tesis de Maestria -con mención en Educación- presentada y defendida por el autor en la FLACSO (sede Argentina), dirigida por la Dra. Mónica de la Fare, bajo el título de "Las apropiaciones y usos intelectuales de la obra de Pierre Bourdieu en el campo educativo argentino (1971-1989)". A lo largo de los tres capítulos que componen el libro, Hernán Amar analiza la producción de conocimiento en el periodo referido, a partir de indagar las recepciones (en términos de usos en la producción concreta de conocimiento) de las obras de Bourdieu, especificamente, en el campo académico educativo. No obstante la acotación espacial y temporal del estudio, la investigación de Amar coloca un problema que debe ser central en el campo de las Ciencias Sociales en la actualidad, especialmente en Latinoamérica: la apropiación (muchas veces no vigilada epistemológicamente) de cuerpos teóricos producidos en marcos espacio-temporales que poco tienen que ver con las realidades regionales y locales.

Amar utiliza las propias herramientas teóricas que el sociólogo francés propuso para el análisis de la producción científica y las pone a prueba en la indagación, como se dijo, de un ámbito particular: el mundo académico vinculado a la educación. Así, considera que este se comporta como un campo científico con "agentes y agencias que toman a la educación como objeto de reflexión desde una perspectiva científica [...] y luchan por detentar el capital científico en sus múltiples formas" (p. 25). A partir de esto, estudia la recepción que las obras de Bourdieu han tenido llevando adelante un exhaustivo relevamiento documental (artículos de revistas científicas de la época, documentos de trabajo de diferentes agencias y libros) y, además, la realización de entrevistas a intelectuales que operaron en esta apropiación intelectual (Emilio Tenti Fanfani y Juan Carlos Tedesco). De este modo, los conceptos de campo científico, capital, habitus, posición y toma de posición le permiten

1 Facultad de Ciencias Humanas de la Universidad Nacional del Centro de la Provincia de Buenos Aires (FCH/UNCPBA), Tandil, BA, Argentina. 
a Hernán Amar reconstruir la complejidad que implicó la recepción de la producción sociológica de Bourdieu en Argentina.

Pierre Bourdieu ha sostenido, respecto de la indagación de los campos científicos, que:

Un análisis que tratara de aislar una dimensión puramente 'política' en los conflictos por la dominación en el campo cientíico sería tan radicalmente falso como su contraparte, más frecuente, el análisis que no considera sino las determinaciones 'puras' y puramente intelectuales de los conflictos cientificos (BOURDIEU, 2008, p. 14).

Estas dimensiones hacen manifiestas dos formas de poder:

por un lado, un poder que podemos llamar temporal (o político), poder institucional e institucionalizado que está ligado a la ocupación de posiciones eminentes en las instituciones científicas [...] y al poder sobre los medios de producción (contratos, créditos, puestos, etc.) y reproducción (facultad de nombrar y promover carreras) que asegura esa posición prominente; por otro lado, un poder especifico, 'prestigio' personal [...] que se basa en el reconocimiento, poco o mal objetivado e institucionalizado, del conjunto de los pares (IBÍD., p. 89).

El estudio de Amar, lejos escindir las dimensiones política e intelectual/científica (o asumir determinaciones lineales entre ambas), evidencia la compleja imbricación entre ellas y, a lo largo de cada uno de los capitulos, muestra cómo se han articulado.

El primer capítulo, La llegada (1971-1976), da cuenta de cómo ya las primeras recepciones de las obras de Pierre Bourdieu constituyeron verdaderas apropiaciones que implicaron la producción de diagnósticos sobre problemáticas educativas a través del uso de categorias conceptuales y esquemas analiticos provenientes, sobre todo, de "La reproducción" (aunque también se realizaron, en el mismo periodo, lecturas en clave epistemológica de "El oficio de sociólogo"). Así, si bien la apropiación fue relativamente parcial (puesto que otros escritos de Bourdieu -algunos traducidos ya en la época-, no fueron considerados con el mismo interés ni como herramientas de análisis del mundo educativo), fue el punto de partida para la conformación de un nuevo habitus científico. Entendido este último como "un 'oficio', es decir, un sentido práctico de los problemas que se van a tratar, unas maneras adecuadas de tratarlos" (Bourdieu, 2003, p. 73), contribuyó, según pudo constatar Hernán Amar, a tomar una posición político-pedagógica que legitimara una visión crítica sobre la relación entre el Estado, la sociedad y la educación, de forma muy diferente a las posturas tecnocráticas imperantes en ese momento.

El segundo capitulo, La circulación soterrada y las voces exiliadas (1976-1983), muestra cómo, en el marco de la dictadura más cruenta que ha tenido Argentina, las lecturas de Bourdieu se hicieron desde organismos regionales e intergubernamentales (CEPAL, FLACSO) y desde México (pais de exilio para muchos y muchas intelectuales). Este contexto posibilitó la profundización de una lectura en clave marxista de la obra de Pierre Bourdieu y, de este modo, se acentuó su dimensión estructuralista. Hernán Amar da cuenta, no obstante estos principios generales, de la ecléctica y heterogénea apropiación intelectual de la profusa y diversa obra de Bourdieu. Tanto en este capitulo como en el anterior, el autor presenta un detallado análisis de los diferentes artículos de revistas científicas, de documentos y de libros en los cuales se evidencian los distintos usos de la arquitectura conceptual del sociólogo francés.

En el tercer capítulo, "Lecturas de auctor" en la democracia recuperada (1983-1989), Amar analiza la producción que "con y contra Bourdieu" (p. 96) realizaron tres referentes intelectuales. Siguiendo la distinción que Bourdieu elaboró entre lectores (simples comentadores) y auctores, "los que leen para hacer algo, para hacer avanzar el conocimiento" (Bourdieu, 2000, p. 198), el autor presenta, asi, el estudio de diferentes producciones que Emilio Tenti Fanfani, Juan Carlos Tedesco y Cecilia Braslavsky elaboraron durante la apertura democrática argentina. Amar da cuenta de cómo este periodo significó una reconfiguración del campo académico educativo en dos sentidos: por un lado, en términos de su relación con el campo de poder, dado que las políticas educativas se 
hicieron eco de las producciones académicas (que mostraban los rasgos autoritarios del sistema educativo, las desigualdades que había en su interior y los efectos reproductores); por otro, en referencia a la propia autonomía del campo, puesto que se impulsó la producción de libros y revistas especializadas y se generaron condiciones para la repatriación de científicos y la vinculación con agentes de campos científicos de otros países. No obstante, señala Hernán Amar, los usos intelectuales quedaron, en su mayoría (con excepciones que el autor se encarga de señalar y analizar), ceñidos todavía a las lecturas de "La reproducción".

\section{Evaluación}

Acaso uno de los postulados epistemológicos más relevantes -y polémicos- a los cuales Pierre Bourdieu ha vuelto una y otra vez a lo largo de su obra se refiere a la necesidad que tiene la comunidad científica de objetivar los procesos de objetivación, en términos de "volverlos la los instrumentos teóricos y técnicos de objetivación], de alguna manera, contra sí mismo, de objetivar su propia posición" (BOURDIEU, 2009, p. 27) proceso bajo el cual debe ser leido, e.g., Homo Academicus, según el propio autor (Cfr. BOURDIEU \& WACQUANT, 2005). Trabajos anteriores al de Amar, como los de Ana Teresa Martinez (2008) -respecto a las lecturas que de Bourdieu se han hecho en Francia y en Estados Unidos-, de Denis Baranger (2008) -para el caso de la recepción argentina y brasileña- y de Afrânio y Denice Catani y Gilson Pereira (2001) -respecto de la recepción brasileña-, retoman esta premisa y van en esta línea de contribuciones: aportan a reflexionar, al tomar por objeto el propio campo científico, cómo se configuran y reconfiguran, en determinados momentos históricos y lugares particulares, las relaciones entre posiciones de agentes que poseen diferenciales volúmenes de capital científico y legitimidad; cómo se vincula el campo con el político y de qué forma se evidencian pujas por la imposición de determinadas tradiciones teóricas.

El trabajo exhaustivo y detallado que Hernán Amar presenta en este libro da cuenta de esta línea de reflexión y, por ende, resulta relevante para las discusiones en el campo de las Ciencias Sociales en nuestra región toda. Contribuye, así, a analizar lo que Hugo Zemelman (2005) ha denominado la "pertinencia histórica del conocimiento" (p. 78), es decir, la capacidad de los conceptos y esquemas analíticos para dar cuenta, efectivamente, de los fenómenos sociales (con las particularidades que Latinoamérica posee). Esta objetivación de los puntos de vista desde los cuales se objetiva el mundo social permite "evitar ser atrapados por las inercias heredadas" (Ibíd.: 122). Inercias que, en el caso de las apropiaciones de la propuesta teórica de Bourdieu, son y han sido tan frecuentes.

\section{REFERENCIAS}

Baranger, D. (2008, Diciembre). La recepción de Bourdieu en Argentina y en Brasil. Trabajo presentado In $V$ Jornadas de Sociología de la Universidad Nacional de La Plata, La Plata, Argentina. Recuperado de https:// www.aacademica.org/000-096/542.pdf.

Bourdieu, P. (2000). Intelectuales, politica y poder. Buenos Aires: EUDEBA.

Bourdieu, P. (2003). El oficio de científico. Ciencia de la ciencia y reflexividad. Curso del College de France 2000-2001. Barcelona: Editorial Anagrama.

Bourdieu, P. (2008). Los usos sociales de la ciencia Buenos Aires: Nueva Visión. (Original publicado en 1997).

Bourdieu, P. (2009). Homo Academicus. México: Siglo XXI. (Original publicado en 1984).

Bourdieu, P. \& Wacquant, L. (2005). Una invitación a la sociología reflexiva. Buenos Aires: Siglo XXI.

Catani, A. M., Catani, D. B., \& Pereira, G. R. de M. (2001). As apropriações da obra de Pierre Bourdieu no campo educacional brasileiro, através de periódicos da área. Revista Brasileira de Educação, 17, 63-85. DOI: https:// doi.org/10.1590/S1413-24782001000200006.

Martinez, A. T. (2008). Lectura y lectores de Pierre Bourdieu. Lecciones y equivocos desde Francia y EE.UU. Cuadernos de la Facultad de Humanidades y Ciencias Sociales- Universidad Nacional de Jujuy, 34, mayo, 251-268. DOI: http://www.scielo.org.ar/pdf/ cfhycs/n34/n34a17.pdf.

Zemelman, H. (2005). Voluntad de conocer. El sujeto y su pensamiento en el paradigma crítico. México: Centro de Investigaciones Humanísticas-Universidad Autónoma de Chiapas 


\section{Iván Surge}

Maestrando en Ciencias Sociales y Humanidades, orientación Sociología, por la Universidad Nacional de Quilmes (UNQ). Prof. en Ciencias de la Educación por la Facultad de Ciencias Humanas de la Universidad Nacional del Centro de la Provincia de Buenos Aires (FCH/UNCPBA), Argentina. Docente e investigador de la misma universidad. Miembro del Núcleo de Investigaciones Educacionales y Sociales (NEES/FCH/UNCPBA).

\section{Dirección}

Iván Surge

Facultad de Ciencias Humanas de la Universidad Nacional del Centro de la Provincia de Buenos Aires

Paraje Arroyo Seco s/n

\section{C.P. B7000GHG}

Tandil, BA, Argentina 